\title{
Combined Nimotuzumab with Chemoradiotherapy for Locally Advanced Head and Neck Squamous Cell Carcinoma
}

\author{
Nguyen Thi Thai Hoa ${ }^{1}$, Huynh Quang Huy ${ }^{2}$ \\ 1. Department of Internal Medicine, Vietnam National Cancer Hospital, Hanoi, VNM 2. Radiology, Pham Ngoc Thach \\ University of Medicine, Ho Chi Minh City, VNM
}

Corresponding author: Huynh Quang Huy, huyhq@pnt.edu.vn

\section{Abstract \\ Background}

Most head and neck cancers (HNCs), specifically squamous cell carcinoma, express epidermal growth factor and are associated with an inadequate response to radiotherapy and chemotherapy. Anti-epidermal growth factor receptor (EGFR) monoclonal antibodies $(\mathrm{mAb})$ increase response rates and survival when combined with radiotherapy or chemoradiotherapy (CRT). This study evaluates the outcome and toxicity of the nimotuzumab-CRT combination for stage III, IVa, and IVb squamous cell carcinoma of the head and neck.

\section{Methods}

Eighty-seven patients with squamous cell carcinoma of the head and neck, stage III, IVa, or IVb were enrolled in a prospective comparative study. The nimotuzumab plus CRT group consisted of patients who received nimotuzumab $200 \mathrm{mg}$ every week for six consecutive weeks chemoradiation therapy; cisplatin 30 $\mathrm{mg} / \mathrm{m}^{2}$ every week for six weeks; radiotherapy of 2-Gy/fraction, five fractions/week for a total dose of $70 \mathrm{~Gy}$; and neck lymph node invasion prophylaxis at $50 \mathrm{~Gy}$. The CRT alone arm was treated with CRT (without nimotuzumab).

\section{Results}

Tumor response rate of 90.6\% was achieved in nimotuzumab plus CRT group (complete response: $58.1 \%$ ), and $70.4 \%$ in CRT alone arm (complete response: $38.6 \%$; $=0.029$ ). The lymph node response rate was $83.4 \%$ in nimotuzumab plus CRT group (complete response: $46.7 \%$ ), and $73.0 \%$ in CRT group (complete response: 23.0\%). The general response rate in nimotuzumab plus CRT group was $86.0 \%$ (complete response: $48.8 \%$ ), and $68.0 \%$ in CRT alone arm (complete response: $36.0 \%$ ). Twelve-month overall survival (OS) was $75.1 \%$ for the nimotuzumab plus CRT group and $54.4 \%$ for the CRT group. The 24 -month survival was $48.0 \%$ (nimotuzumab plus CRT group) and 29.0\% (CRT alone arm). The median OS was 20 months and 13 months for nimotuzumab plus CRT group and CRT alone arm, respectively. Progression-free survival (PFS) in the nimotuzumab plus CRT group at 12 months and 24 months was $64.2 \%$ and $37.4 \%$, respectively. PFS in the CRT group at 12 months and 24 months was $39.5 \%$ and $21.3 \%$, respectively. Infusion reaction presented mildly in two of 43 patients in the nimotuzumab plus CRT group, and no shock occurred. Other toxicity occurrences were similar between the two groups, mainly in grade I, II. Skin rash (grade I only) occurred at a rate of $4.7 \%$ in the nimotuzumab plus CRT group.

\section{Conclusion}

Nimotuzumab in combination with CRT was well tolerated as a treatment program for locally advanced head and neck squamous cell carcinoma.

Categories: Oncology

Keywords: monoclonnal antibody, chemoradiotherapy, response rate, overall survival, progression - free survival

\section{Introduction}

Head and neck cancer (HNC) is a group of cancers derived from different locations in the upper respiratory and digestive tracts. HNCs account for $10 \%$ of all types of cancer. Most malignant tumors of the head and neck are derived from epithelial surfaces, so more than $90 \%$ of cases are usually accounted for by squamous cell carcinoma or its variants. Management of HNC depends on the anatomical location and stage of the disease. In the case of locally advanced disease, chemoradiation therapy with cisplatin gives good results under appropriate indications [1].

More than $95 \%$ of HNCs, especially squamous cell carcinoma, express epidermal growth factor, and are associated with a poor response to radiotherapy and chemotherapy [2]. Anti-epidermal growth factor 
receptor (EGFR) monoclonal antibodies $(\mathrm{mAb})$ increase response rates and survival when combined with radiotherapy or chemoradiotherapy(CRT) [3-6].

In Vietnam, the anti-EGFR mAb nimotuzumab has been used in combination with CRT or radiotherapy for the treatment of HNC since 2009; so far, no studies have addressed the effectiveness and safety of this regimen. This study evaluates the outcome and toxicity of the nimotuzumab-CRT combination for stage III, IVa, and IVb squamous cell carcinoma of the head and neck.

\section{Materials And Methods \\ Patients}

Patients diagnosed with squamous cell carcinoma of the head and neck with the American Joint Committee on Cancer (AJCC) stage III, IVa, or IVb were enrolled in a prospective comparative study [7]. This study was conducted at Vietnam National Cancer Hospital from June 2010 to June 2013 under the approval of the Hospital Ethics Committee (reference number: 01062010/VNCH). Written informed consent was obtained from all patients before inclusion in the study.

Inclusion criteria were as follows: squamous cell carcinoma of the oral cavity, oral pharynx, hypopharynx and larynx (stage III, IVA, or IVB) according to the AJCC; age older than 18 years; first-time treatment; performance status (PS) of zero to two; provided informed consent to participate in the study [7].

Exclusion criteria were as follows: cancer of the salivary glands, sinuses, nasopharynx, skin, or lips; indication for radical surgery; a history of other cancers; a history of chemotherapy, radiotherapy or antiEGFR treatment; a history of severe chronic diseases (e.g., diabetes, hypertension, HIV, viral hepatitis) resulting in contraindication for chemotherapy or anti-EGFR $\mathrm{mAb}$; and pregnant or breastfeeding women.

Variables of patients were collected, including diagnosis, age, gender, tumor stage, tumor grade according to World Health Organization classification of tumors, PS based on The Eastern Cooperative Oncology Group (ECOG) score, investigations, clinical course, with details of concurrent chemotherapy, radiotherapy, nimotuzumab therapy $[8,9]$.

\section{Treatment protocol}

Two treatment group were defined. Patients were randomized to receive the treatment by simple randomization method.

Nimotuzumab plus CRT group consisted of patients who received nimotuzumab chemoradiation therapy as follows: nimotuzumab $200 \mathrm{mg}$ every week for six consecutive weeks; cisplatin $30 \mathrm{mg} / \mathrm{m} 2$ every week for six weeks; radiotherapy of 2-Gy/fraction, five fractions/week for a total dose of $70 \mathrm{~Gy}$; neck lymph node invasion prophylaxis of $50 \mathrm{~Gy}$. CRT group was treated with CRT (without nimotuzumab).

\section{Follow-up during and after treatment}

The treatment response was evaluated at eight weeks following radiotherapy using Response Evaluation Criteria in Solid Tumors version 1.0 [10]. The responses assessed included complete response (CR), partial response (PR), the progression of disease (PD), and stable disease (SD) based on computed tomography (CT) findings.

Evaluation of side effects was performed according to Common Terminology Criteria Standard for Adverse Events version 3.0 [11]. Patients were followed up for at least two years or death to assess survival time.

\section{Statistical analysis}

Data were processed using Statistical Package for the Social Sciences for Windows, Version 16.0. (SPSS Inc., Chicago, IL). Progression-free survival (PFS) and overall survival (OS) were measured using the KaplanMeier method from the beginning of treatment until the date of death or last date of follow-up. Univariate analysis of patient characteristics and tumor response was conducted by chi-square test and Fisher's exact test. All probability values were two-sided, and $\mathrm{P}$ values $<0.05$ were considered statistically significant.

\section{Results}

A total of 87 patients were included in the study; 43 patients were in the nimotuzumab plus CRT group and 44 in the CRT alone arm. Table 1 summarizes the characteristics of 87 patients included in the analysis. There was no significant difference between the two groups in terms of age, gender, tumor location, tumor/node/metastasis stage, grade, and PS of patients. 


\section{Cureus}

Age group (n, \%)

30-39 years

4 (9.3)

2 (4.5)

40-49 years

9 (20.9)

9 (20.5)

50-59 years

21 (48.0)

25 (56.8)

$\geq 60$ years

9 (20.9)

8 (18.2)

Gender (n, \%)

Male

40 (93.0)

42 (95.5)

Female

$3(7.0)$

2 (4.5)

Location $(\mathrm{n}, \%)$

Oral cavity

$11(25.5)$

15 (34.1)

Oropharynx

10 (23.3)

8 (18.1)

Hypopharynx, larynx

22 (51.2)

21 (47.8)

T-stage (n,\%)

1

$0(0.0)$

$0(0.0)$

2

$1(2.3)$

$1(2.3)$

3

$13(30.2)$

16 (36.4)

$4 \mathrm{~A}$

20 (46.5)

20 (45.5)

4B

9 (20.9)

7 (15.9)

N-stage (n, \%)

No

$13(30.2)$

16 (36.4)

12 (27.9)

12 (27.3)

12 (27.9)

$12(27.3)$

6 (14.0)

4 (9.1)

Overall Stage (n, \%)

III

7 (16.3)

$14(31.8)$

IVa

22 (51.1)

19 (43.2)

IVb

14 (32.6)

$11(25.0)$

Grade

I

6 (14.0)

6 (13.6)

II

29 (67.4)

34 (77.3)

III

8 (18.6)

$4(9.1)$

Performance status (PS) (n, \%)

0

$26(60.5)$

$21(47.7)$

1

13 (30.2)

18 (40.9)

2

4 (9.3)

5 (11.4)

TABLE 1: Patient characteristics

CRT: chemoradiotherapy. 


\section{Cureus}

The complete and partial response rates of nimotuzumab plus CRT group were $48.8 \%$ and $37.2 \%$, respectively, compared to the $34.1 \%$ and $36.4 \%$ complete and partial response rates (respectively) in CRT group. There was no significant difference between the two groups (Table 2).

The overall tumor response rate across both groups was $80.5 \%$ (with $48.3 \%$ complete response and $32.2 \%$ partial response). The tumor response rate in the nimotuzumab plus CRT group and CRT alone arm was $90.6 \%$ and $70.4 \%$, respectively; the difference was statistically significant $(\mathrm{p}=0.029)$. Overall, the lymph node response across both groups was $82.8 \%$ (39.7\% complete response and $43.1 \%$ partial response). The general response in the two groups was $78.2 \%$, with $41.4 \%$ complete response and a $36.8 \%$ partial response (Table 2).

\begin{tabular}{|c|c|c|}
\hline Response & Nimotuzumab plus CRT groups & CRT alone group \\
\hline \multicolumn{3}{|l|}{ Tumor (n,\%) } \\
\hline Complete & $25(58.1)$ & $17(38.6)$ \\
\hline Partial & $14(32.5)$ & $14(31.8)$ \\
\hline No response & $2(4.7)$ & 9 (20.5) \\
\hline Progress & $2(4.7)$ & $4(9.1)$ \\
\hline \multicolumn{3}{|c|}{ Lymph nodes (n,\%) } \\
\hline Complete & $14(46.7)$ & $9(32.1)$ \\
\hline Partial & $11(36.7)$ & $14(50.0)$ \\
\hline No response & $2(6.6)$ & $2(7.1)$ \\
\hline Progress & $3(10.0)$ & $3(10.8)$ \\
\hline \multicolumn{3}{|c|}{ General response $(n, \%)$} \\
\hline Complete & $21(48.8)$ & $15(34.1)$ \\
\hline Partial & $16(37.2)$ & $16(36.4)$ \\
\hline No response & $4(9.3)$ & 6 (13.6) \\
\hline Progress & $2(4.7)$ & $7(15.9)$ \\
\hline
\end{tabular}

TABLE 2: Response rate within the two groups

CRT: chemoradiotherapy.

OS in the two groups at 12 and 24 months was $75.1 \%$ and $48.0 \%$, respectively, in the nimotuzumab plus CRT group and $54.4 \%$ and $29.0 \%$ in CRT group; the difference was statistically significant $(\mathrm{p}=0.016)$ (Figure 1$)$. 


\section{Cureus}

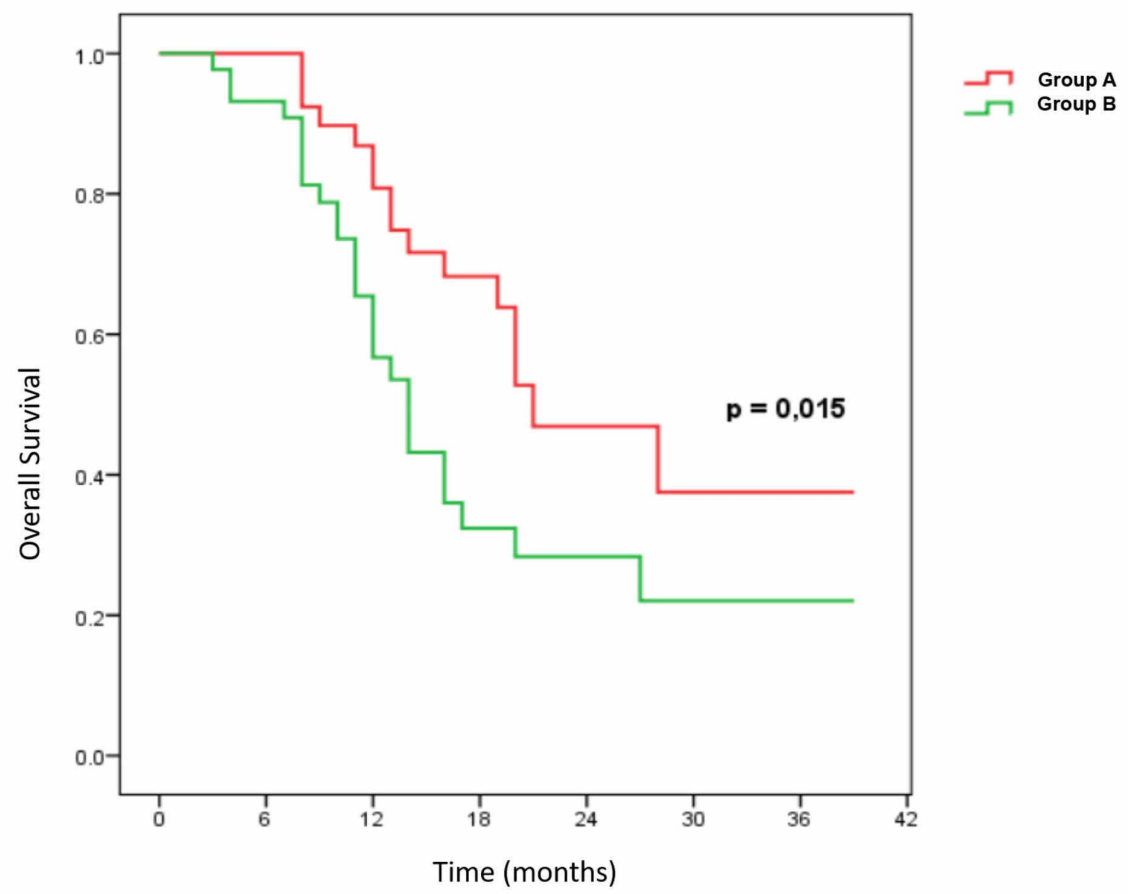

FIGURE 1: Overall survival of two groups

Group A: Nimotuzumab plus chemoradiotherapy (CRT); Group B: CRT alone.

Multivariable analysis identified lymph node metastasis as a risk factor affecting treatment outcomes when combining monoclonal antibody with chemoradiation therapy.

PFS of the two groups at 12 and 24 months was $64.2 \%$ and $37.4 \%$, respectively, in the nimotuzumab plus CRT group compared to $39.5 \%$ and $21.3 \%$ in the CRT group. The difference was statistically significant $(\mathrm{p}=0.016)$ (Figure 2).

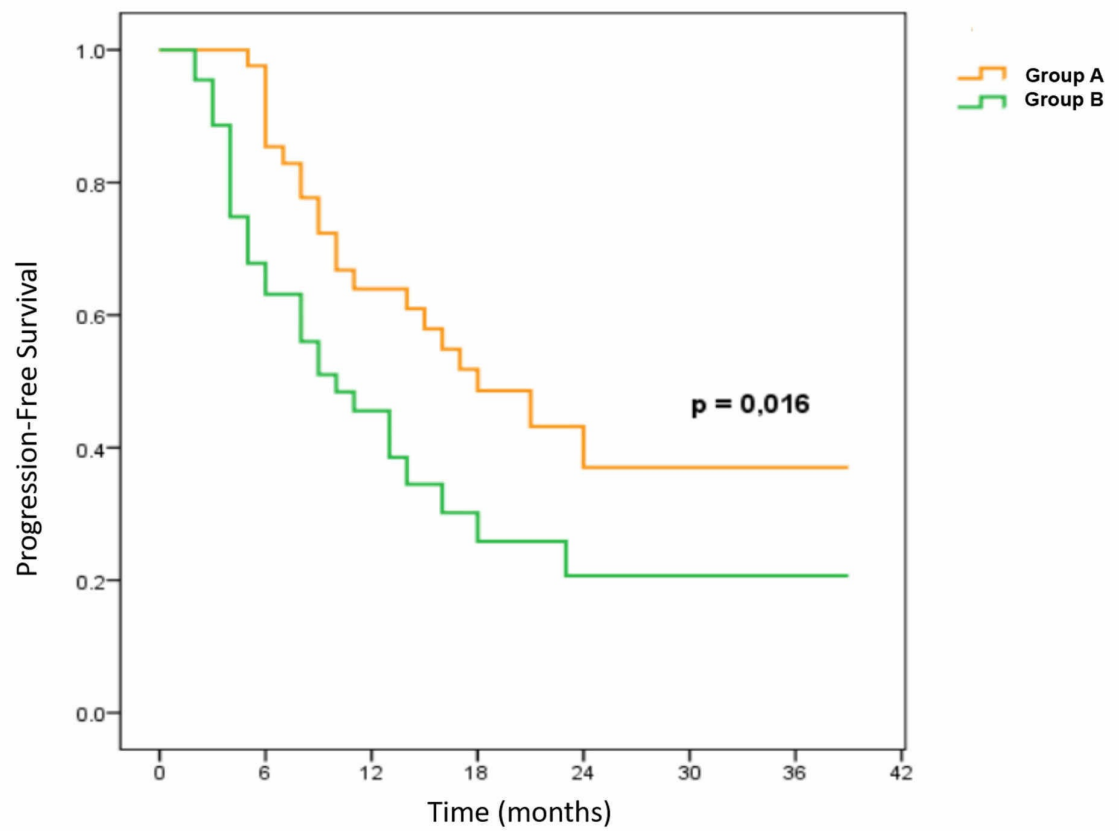

FIGURE 2: Progression-free survival of two groups

Group A: Nimotuzumab plus chemoradiotherapy (CRT); Group B: CRT alone. 


\section{Cureus}

The most common adverse events encountered during treatment and detailed toxicity and grade for the combination therapy are summarized in Table 3 and Table 4. Although these adverse events slightly delayed the treatment for several weeks, no patients halted therapy or died (Table 5).

\begin{tabular}{|l|l|l|}
\hline Side effects & Nimotuzumab plus CRT group (Number of patients) & CRT alone group (Number of patients) \\
\hline Headache & 2 & 0 \\
Flushing & 2 & 0 \\
Itching & 2 & 0 \\
Myalgia & 0 & 0 \\
Hypotension & 0 & 0 \\
Chills & 0 & 0 \\
Fever & 0 & 0 \\
Shock & 0 & 44 \\
Total & 43 & 0 \\
\hline
\end{tabular}

\section{TABLE 3: Infusion reactions}

CRT: chemoradiotherapy.

\begin{tabular}{|c|c|c|c|c|c|c|}
\hline \multirow{2}{*}{ Side Effects } & \multicolumn{3}{|c|}{ Stage I, II, III, IV } & \multicolumn{3}{|l|}{ Stage III, IV } \\
\hline & Group A (\%) & Group B (\%) & $p$ & Group A (\%) & Group B (\%) & $\mathrm{p}$ \\
\hline Neutropenia & $9(21.0)$ & $14(31.8)$ & $>0.05$ & $0(0.0)$ & $0(0.0)$ & - \\
\hline Anemia & $13(30.2)$ & 17 (38.7) & $>0.05$ & $0(0.0)$ & $1(2.3)$ & - \\
\hline Thrombocytopenia & $1(2.3)$ & $3(6.8)$ & - & $0(0.0)$ & $0(0.0)$ & - \\
\hline Nausea & $16(37.2)$ & $16(36.4)$ & $>0.05$ & $0(0.0)$ & $1(2.3)$ & - \\
\hline Vomiting & 17 (39.5) & $20(45.5)$ & $>0.05$ & $0(0.0)$ & $1(2.3)$ & - \\
\hline Renal failure & & & & $1(2.3)$ & $0(0.0)$ & - \\
\hline LFT elevations & $5(11.6)$ & $3(6.8)$ & - & $0(0.0)$ & $0(0.0)$ & - \\
\hline Mucositis & 38 (88.4) & 39 (88.6) & $>0.05$ & $11(25.6)$ & $9(20.5)$ & $>0.05$ \\
\hline Dermatitis & $26(60.5)$ & $22(50.0)$ & $>0.05$ & $1(2.3)$ & $0(0.0)$ & - \\
\hline Rash & $2(4.7)$ & $0(0.0)$ & - & $0(0.0)$ & $0(0.0)$ & - \\
\hline
\end{tabular}

\section{TABLE 4: Other adverse events}

Group A: Nimotuzumab plus chemoradiotherapy (CRT); Group B: CRT alone; LFT: liver function test. 


\section{Cureus}

\begin{tabular}{|c|c|c|}
\hline Delay time & Nimotuzumab plus CRT group $(n, \%)$ & CRT alone group $(n, \%)$ \\
\hline$<1$ week & $37(86.0)$ & $40(90.1)$ \\
\hline 1-2 weeks & $4(9.3)$ & $4(9.1)$ \\
\hline 2-3 weeks & $2(4.6)$ & $0(0)$ \\
\hline Stop treatment & 0 & 0 \\
\hline Death & 0 & 0 \\
\hline Total & 43 & 44 \\
\hline
\end{tabular}

TABLE 5: Treatment delay due to side effects

CRT: chemoradiotherapy.

\section{Discussion}

We saw an overall tumor response rate across both groups of $80.5 \%$, with $48.3 \%$ complete response and $32.2 \%$ partial response. Ramakrishnan et al. conducted a study among 40 patients treated with CRT with or without nimotuzumab, and they reported 34 cases had partial or complete response accounting for $85 \%$, with complete response in 32 cases accounting for $80 \%$ of the cohort [5]. Their response rate was slightly higher than ours, with an obvious difference in the complete response rate. The difference in response rates may be because, in this study, the authors only took phase III, IVa (T1-4a, N0-2) and excluded T4B, N3, which constitute large and invasive tumors that spread, or indicates lymph nodes larger than $6 \mathrm{~cm}$, thus signifying a difficult response to radiotherapy or CRT.

Several domestic studies also mentioned the response rate to CRT but only for specific tumor locations such as the lower pharynx larynx, or tonsils. According to Tung, tumor response rates were as high as $100 \%$ (complete and partial responses were $71.7 \%$ and $28.3 \%$ respectively), and lymph node response was also higher than in our study at $95.4 \%$ (62.8\% complete and 32.6\% partial response) (Ph.D. Thesis: Ngo Thanh Tung: Study on Clinical, Subclinical Characteristics and Results Chemistry - Accelerated Radiotherapy and Laryngeal Cancer in Stage (III - IVB) Cannot Be Operated at Vietnam Cancer Hospital. Ha Noi Medical University. 2011). In that study, response assessment was conducted immediately after treatment, with follow-up for four weeks after the end of treatment. In our study, the assessment was performed later in order to fully evaluate the effectiveness of radiotherapy. According to Hoang et al., tonsillar cancer had a high response after CRT with $80 \%$ partial and 19\% complete response [12]. While this could be explained by the fact that in this study, patients were mainly in stage III or IVa, tonsil carcinoma is still considered to have good treatment outcomes among head and neck squamous cell carcinomas.

The anti-EGFR mAb incorporated into radiotherapy increased response rates across multiple studies. According to Rodríguez, the complete response among patients treated with nimotuzumab combined with radiotherapy was $59.5 \%$, compared with $34.2 \%$ for radiotherapy-placebo. The difference was statistically significant $(\mathrm{p}=0.028)$, which showed that the combination with nimotuzumab had a better response than isolated radiotherapy [6]. Similarly, in the study by Ramakrishnan et al., nimotuzumab increased complete response significantly when combined with radiotherapy (76\% versus $37 \%, p=0.023)$ [5].

Nimotuzumab also increased response rates when combined with CRT, as reported by Ramakrishnan et al. With 20 patients in each group, when combining with nimotuzumab, total response (including complete response and partial response) rose to $100 \%$, compared with $70 \%$ in the group treated with radiotherapy alone $(p=0.02)$ [5]. Similarly, Patil et al. demonstrated that nimotuzumab in combination with cisplatin and radiotherapy was superior to cisplatin and radiotherapy in improving the PFS, duration of locoregional control and disease-free survival in locally advanced squamous head and neck cancer [13].

In our study, the tumor response rate in nimotuzumab plus CRT group and CRT alone arm was $90.6 \%$ and $70.4 \%$, respectively; the difference was statistically significant $(\mathrm{p}=0.029)$. The lymph node response rate and general response rate were higher in the nimotuzumab plus CRT group compared with the CRT group, but the difference was not statistically significant. The complete response rate was higher in the nimotuzumab plus CRT group, but the difference was not statistically significant. Because the number of patients in the study was limited, early results will need to be confirmed by further studies with larger sample sizes.

According to research results, the advantage observed in OS confirmed the benefits of anti-EGFR mAb combination. Specifically, OS at 12 months and 24 months was $75.1 \%$ and $48 \%$ in nimotuzumab plus CRT 
group respectively compared with $54.4 \%$ and $29 \%$ in CRT group; median survival was 20 months in nimotuzumab plus CRT group and 13 months in CRT group, a statistically significant difference $(\mathrm{p}=0.015)$. This result was reproduced for PFS. The rate of PFS in the two groups at 12 and 24 months was $64.2 \%$ and $37.4 \%$ respectively in the nimotuzumab plus CRT group compared to $39.5 \%$ and $21.3 \%$ in CRT group; the difference between the two groups was statistically significant $(\mathrm{p}=0.016)$.

Several large clinical trials have shown the effectiveness of anti-EGFR mAb in combination with radiotherapy or standard CRT. According to Bonner et al., cetuximab in combination with radiotherapy increased median OS from 29.3 to 49 months, decreased mortality rate by $27 \%(p=0.018)$, total five-year survival from $36.4 \%$ to $45.6 \%$, median PFS from 12.4 months to 17.1 months ( $\mathrm{p}=0.006$ ); PFS at two years increased from $37 \%$ to $46 \%$ [3]. Rodriguez et al., when incorporating mAb nimotuzumab into radiotherapy, also found similar results with OS (mean and median) in the antibody nimotuzumab plus CRT group at 21.71 and 12.5 months versus placebo at 17.71 and 9.47 months [6]. This further supports the efficacy of the antiEGFR mAb combination.

For anti-EGFR mAb combination with CRT, previous research has shown results consistent with ours, as demonstrated in a Phase II study by Ramakrishnan et al. showing two-year survival of 39.13\% in the control group and $76.28 \%$ in the combination therapy group $(\mathrm{p}=0.007)$. Mean survival was increased from 23.69 to 43.62 months when combining the treatment with antibodies [5]. Since patients with stage IVb disease were excluded, this study showed higher survival. However, the role of nimotuzumab in combination with standard CRT was also confirmed, similar to our study.

However, the effectiveness of anti-EGFR mAb CRT combination was not confirmed in a 40-month study by Ang et al., wherein 895 patients were evaluated, and initial results showed no difference in OS and PFS [14].

Hematopoietic system toxicity included neutropenia, anemia, thrombocytopenia, mainly at the level I and II. Anemia was most common (30.2\% in nimotuzumab plus CRT group and 38.7\% in CRT group). Only $2.3 \%$ of patients in the CRT group had anemia grade III. Neutropenia was only recorded in grade I and II and occurred in $21 \%$ of patients in the nimotuzumab plus CRT group and $31.8 \%$ in the CRT group.

Thrombocytopenia was rare, occurring at a rate of $2.3 \%$ in the nimotuzumab plus CRT group and $6.8 \%$ in the CRT group. Hematological toxicity in the CRT group was most frequently observed in grade II, and anemia was the most common finding in our study and others $[15,16]$. In the study Tung, using cisplatin $100 \mathrm{mg} / \mathrm{m} 2$ every three weeks, the rate of hematopoietic system toxicity was higher than that observed in our study. However, the severity was relatively similar (Ph.D. Thesis: Tung, 2011).

The rate of vomiting in our study was lower than that reported by Hoang, with $51.5 \%$ of patients experiencing vomiting, the majority of which was grade I (45.3\%), while grade II accounted for only $6.2 \%$. The high rate of vomiting in this study may be due to high dose cisplatin (100 mg/m2 every three weeks) [12]. Although cisplatin is nephrotoxic, with low-dose weekly use, renal failure was rare and mild in our experience. Only one case of renal failure (grade III) was noted, accounting for $2.3 \%$ of patients in the CRT group; the toxicity was reversible, and the resulting treatment delay was minimal.

The infusion reaction associated with mAb nimotuzumab may occur in a small percentage of patients and can usually be fully controlled. According to Crombet et al., this undesirable effect occurs immediately after the administration of nimotuzumab [4]. Patients may experience fever, nausea, vomiting, high blood pressure, chills, headache, disorientation, chest pain, speech disturbances, and/or muscle aches. In our study, hypersensitivity reactions were infrequent, occurring in only two of 43 cases in the nimotuzumab plus CRT group and manifesting as headache, flushing, and itching (accounting for $4.7 \%$ for each type of effect). No cases of anaphylactic shock during infusion were reported. These results are in line with the conclusions of previous studies on the safety of nimotuzumab.

In our study, skin rash occurred in only two of the 43 patients in the nimotuzumab plus CRT group, accounting for $4.7 \%$. Both reactions were rated as grade I and were controlled with a topical cream, without disturbing the course of therapy. This result was consistent with other studies using nimotuzumab.

\section{Conclusions}

Nimotuzumab can be safely combined with chemoradiation therapy for locally advanced squamous cell carcinoma of the head and neck in order to achieve superior therapeutic response and improved survival outcomes without increased toxicity.

\section{Additional Information \\ Disclosures}

Human subjects: Consent was obtained by all participants in this study. Research Ethics Committee of Vietnam National Cancer Hospital issued approval 01062010/VNCH. Animal subjects: All authors have confirmed that this study did not involve animal subjects or tissue. Conflicts of interest: In compliance with the ICMJE uniform disclosure form, all authors declare the following: Payment/services info: All 
authors have declared that no financial support was received from any organization for the submitted work. Financial relationships: All authors have declared that they have no financial relationships at present or within the previous three years with any organizations that might have an interest in the submitted work.

Other relationships: All authors have declared that there are no other relationships or activities that could appear to have influenced the submitted work.

\section{References}

1. Pignon JP, le Maitre A, Maillard E, Bourhis J: Meta-analysis of chemotherapy in head and neck cancer (MACH-NC): an update on 93 randomised trials and 17,346 patients. Radiother Oncol. 2009, 92:4-14. 10.1016/j.radonc.2009.04.014

2. Grandis JR, Melhem MF, Gooding WE, et al.: Levels of TGF-alpha and EGFR protein in head and neck squamous cell carcinoma and patient survival. J Natl Cancer Inst. 1998, 90:824-32. 10.1093/jnci/90.11.824

3. Bonner JA, Harari PM, Giralt J, et al.: Radiotherapy plus cetuximab for locoregionally advanced head and neck cancer: 5-year survival data from a phase 3 randomised trial, and relation between cetuximab-induced rash and survival. Lancet Oncol. 2010, 11:21-8. 10.1016/S1470-2045(09)70311-0

4. Crombet T, Osorio M, Cruz T, et al.: Use of the humanized anti-epidermal growth factor receptor monoclonal antibody h-R3 in combination with radiotherapy in the treatment of locally advanced head and neck cancer patients. J Clin Oncol. 2004, 22:1646-54. 10.1200/JCO.2004.03.089

5. Ramakrishnan MS, Eswaraiah A, Crombet T, Piedra P, Saurez G, Iyer H, Arvind AS: Nimotuzumab, a promising therapeutic monoclonal for treatment of tumors of epithelial origin. mAbs. 2009, 1:41-8. 10.4161/mabs.1.1.7509

6. Rodriguez MO, Rivero TC, del Castillo Bahi R, et al.: Nimotuzumab plus radiotherapy for unresectable squamous-cell carcinoma of the head and neck. Cancer Biol Ther. 2010, 9:343-9. 10.4161/cbt.9.5.10981

7. American Joint Committee on Cancer: AJCC Cancer Staging Manual, Sixth edition. Springer, New York; 2002.

8. Thompson L: World Health Organization classification of tumours: pathology and genetics of head and neck tumours. Ear Nose Throat J. 2006, 85:74.

9. Prigerson HG, Bao Y, Shah MA, et al.: Chemotherapy use, performance status, and quality of life at the end of life. JAMA Oncol. 2015, 1:778-784. 10.1001/jamaoncol.2015.2378

10. Tsuchida Y, Therasse P: Response evaluation criteria in solid tumors (RECIST): new guidelines . Med Pediatr Oncol. 2001, 37:1-3. 10.1002/mpo.1154

11. Zhang S, Chen Q, Wang Q: The use of and adherence to CTCAE v3.0 in cancer clinical trial publications Oncotarget. 2016, 7:65577-65588. 10.18632/oncotarget.11576

12. Hoang LD, Thinh DHQ, Anh CTT: Simultaneous radiation therapy of tonsil carcinoma in advanced stage . Ho Chi Minh City Journal of Medicine. 2010, 150:159.

13. Patil VM, Noronha V, Joshi A: Results of a randomized phase III study of nimotuzumab in combination with concurrent radiotherapy and cisplatin versus radiotherapy and cisplatin alone, in locally advanced squamous cell carcinoma of the head and neck. J Clin Oncol. 2018, 36:6000. 10.1200/JCO.2018.36.15_suppl.6000

14. Ang KK, Zhang Q, Rosenthal DI, et al.: Randomized phase III trial of concurrent accelerated radiation plus cisplatin with or without cetuximab for stage III to IV head and neck carcinoma: RTOG 0522. J Clin Oncol. 2014, 32:2940-50. 10.1200/JCO.2013.53.5633

15. Guo JH, Chen MQ, Chen C, Lu HJ, Xu BH: Efficacy and toxicity of nimotuzumab combined with radiotherapy in elderly patients with esophageal squamous cell carcinoma. Mol Clin Oncol. 2015, 3:1135-1138. $10.3892 /$ mco.2015.606

16. Li HM, Li P, Qian YJ, et al.: A retrospective paired study: efficacy and toxicity of nimotuzumab versus cisplatin concurrent with radiotherapy in nasopharyngeal carcinoma. BMC Cancer. 2016, 16:946. 10.1186/s12885-016-2974-X 